\title{
Analysis of the Multidimensional Space form and Expression Pattern of Book Design
}

\author{
Genqi $L i^{1, a}$ \\ ${ }^{1}$ Zhengzhou University of Industrial Technology, Zhengzhou, Henan, 451100 \\ a email
}

Keywords: Book Design, Multidimensional Space, Space Form

\begin{abstract}
Life books and the people closely related to the book is an example of human learning, a broad sense is the ability to show the crystallization of human civilization history and culture; from the narrow sense to let people learn from previous experience. With the development of the times, people look for books, content layout, paper requirements continue to increase, the design of the book became inevitable product of the times, science and technology can now be produced at the rhythm of the times for the book product, the trend now is an important development. Science and technology continue to progress, and promote a lot of digital technology, photography technology matures, so that we have entered the information age, how to make books designed to keep pace with the times, the goal now is a mature technology, important ideas. Modern books not just the appearance of packaging, or in the books as the carrier, a full range of packaging, from content to form, from structure to form, from flat to three-dimensional space print space, from production to sales of full exhibition space overall design. We can see the "multidimensional space" concept has become a modern book design packaging system is an important dimension. In this paper, the concept of book design multidimensional space form and manifestations, the importance, combining practical now to analyze.
\end{abstract}

\section{Introduction}

Design belongs to the category of art and design books, people can not live without books, from primary school textbooks to people entertainment books, books become necessities of people's lives, but how to make the books suitable for the present pace of life, this is worth considering important issues. The book is designed to read and learn exist to serve the reader's reading experience. Way of being multidimensional space form book designed to show the contents of the range performance is somewhat different, but the ultimate aim is to make the promotion of books in the crowd. Book design multidimensional space form and manifestations is the trend now book cultural development.

\section{The Meaning of Book Design}

Book design concept with the development of society transformed from the beginning, the main purpose is to design book covers, book font design within the design and layout of the book illustrations and materials, make a comprehensive design and processing, in order to see presents the overall image of the book. Book design is a product of the times, it is to design and print production processes combining crystallization.

The ultimate goal is to promote books and Design in the popularity of books to give public recognition, presenting books which artistic content, showing perfect side.

Books in multidimensional space form design to design a lot of respect, it is important is to show a sense of the picture, which will help the public to accept, so that the whole culture connotation of cultural change presented specific connotation, demonstrating the perfect book reading experience.

Book design focus is the human cultural factors, only the public accept the product, is the best use of cultural products and services. Book design includes user-friendly design, only the combination of the design is the best design of the human factors of product, to be able to get public recognition, in order to achieve the best cultural connotation, showing the most outstanding user 
experience.

\section{The Form of Books Multidimensional Space and Manifestation}

Shape books multidimensional space of meaning. Many forms of representation of space books are divided into two aspects, one is the book design appearance of the packaging, the main focus of the book cover packaging, the design is an important reason for the important transition in the contents above; on the other hand in-house version of the book design located on the hardness of the paper, font size, etc., are books inside the packaging design.

Caused by images, text, color fusion good visual experience, get support from readers and showcase for other books is not the same advantages, the display content is most important is to show a wide range of factors can be achieved factors critical issues.

The importance of books multidimensional space. With the development of society, the accelerated pace of urban life, multi-dimensional spatial form and manifestation of books must be able to promote the development of the course books extent, so that books fit people's lives become the target of a paradigm shift now important developments one. Books are the spiritual food, from books only learn more knowledge in order to hold up a person's dream, to show their talent. Multidimensional space form book design from the physiological characteristics of the human viewpoint, is to avoid people appear visual fatigue, conducive to the books of knowledge and understanding; visual learning aspects of people's point of view, book design multidimensional space form able to attract readers eyes, to show the other side of books, increase interest in the sense of books.

Forms, books multidimensional space. By reference to modern elements to attract the reader's eye. Continuous social progress brought about by the above technology revolution, compared to the traditional book conceptual design, book design is now more closer to the pace. Books in use graphics, color and text are to achieve humane, in order to attract the reader's eye. Book design of the main elements is the graphics, colors and text. Designers need to win the reader's eye, we need to start with this one aspect. Book design with the use of multidimensional space form, in which the integration of the three elements, demonstrating the unique aspect of the book.

Point, line, surface is a basic element of the multidimensional space of the book design configuration. Suitable fusion point line and plane, it is possible to show the unique style to attract the reader's eye, unlike other books produced visual experience. Meet the aesthetic ideas of the people to some extent. Constitute a colorful, unique in the world, get a good reading experience.

Color has a head start effect, color constituting the above overall image of book design occupies an important position. Colors give people visual impact of the above, it is possible to bring people of different visual experience. In the book design to fully take into account the layout of the entire writing. Color sense of shock, can cause potential readers the impression that to a certain extent.

Text is the key to the whole book composed. Each character has a charm which, each font has a connotation different from other cultures. So when the book design, take full account of the contents of the article and binding books. Multidimensional space form of books need to consider many factors, not just one aspect of care which. Such as "Divine Comedy" is not designed to deliberately depict specific image of the poet Dante, are not meant to show cause and effect of good and evil poems, but a metaphor for heaven and hell through the black and white color transitions and highly decorative stars, good and evil is the unity of opposites relationship between black and white, between God and man's right and wrong.

The book design to show people and cultural factors, to deepen the emotional content of the text. Books show content is often poured of emotions, the visual impact is the most direct experience of the reader reading. The contents of the book design above, must pay attention to clear and smooth forum, read no obstacle. The articles and content elements combine whole article, creating a warm and have human feelings reading atmosphere. Allows the reader during reading, go inside the author's ideas, which will help understand the contents of the article.

Layout design books for readers, having the key role on the books to show the personality and take into account the needs of the public, to help promote books to some extent in the human design. 
User-friendly design books, the layout design is necessary to demonstrate the art of tension, but also fully embodies the connotation of the original, so that readers in reading as in the music, feel the book unique rhythm and rhyme; even in the page, cross-page fold-page, single-page, and to maintain a certain sense of overall coherence, expand the design in the form of a unified framework to control the visual layout of the order to deepen the reader's emotions through humane layout. Shown in figure II, is displayed by the layout design books, get a different reading experience, compared to traditional books designed to attract readers to a certain extent, of interest to the reader reading.

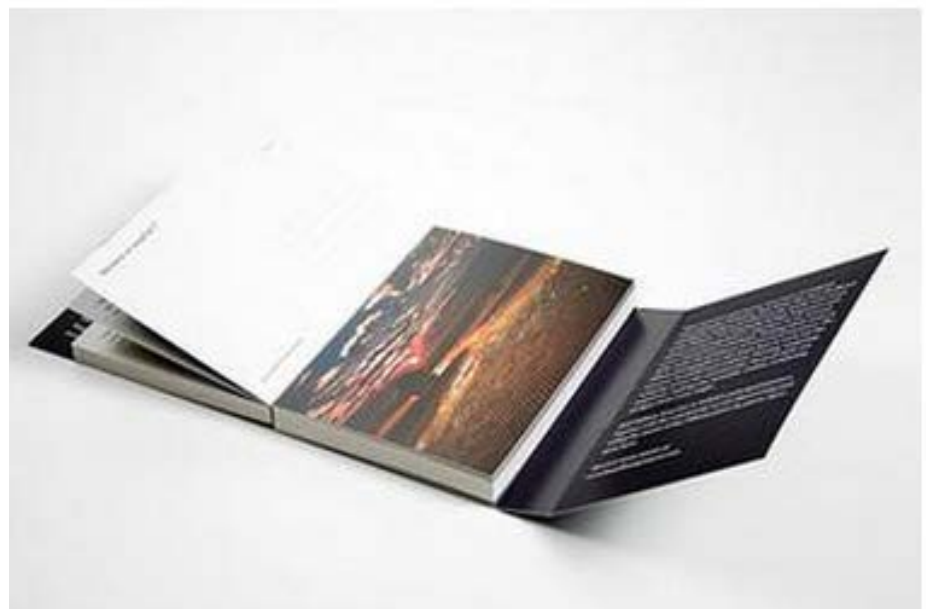

Into the picture elements to focus the reader Perspective. Books insert pictures is now one of the most common artistic expression, can show important factor in the overall cultural phenomenon of change.

Pictures of the insert is divided into two, an art illustrations, also known as literary illustrations, it has the character corresponding to the value of independent views, you can increase the reader's interest in reading some extent; another aspect is the technology illustration, science and technology, also known as illustrations, illustrations of this class, the main aspects of performance is not exhaustive text books represented.

Insert images must fit the contents of the article, to avoid one important reason for the different cultures of the above changes, important cultural differences presented above.

Good illustration can help certain extent, the author of books to attract capital, to reflect the article interesting, academic. Enrich the connotation of the text of the article, adding interest in reading. Insert chart three pictures, the reader is brought back interest in reading, the whole plate Wenzhan show coordination can be compared to a certain extent. And insert images of vivid, help the reader to understand the content of the article, efforts to deepen learning.

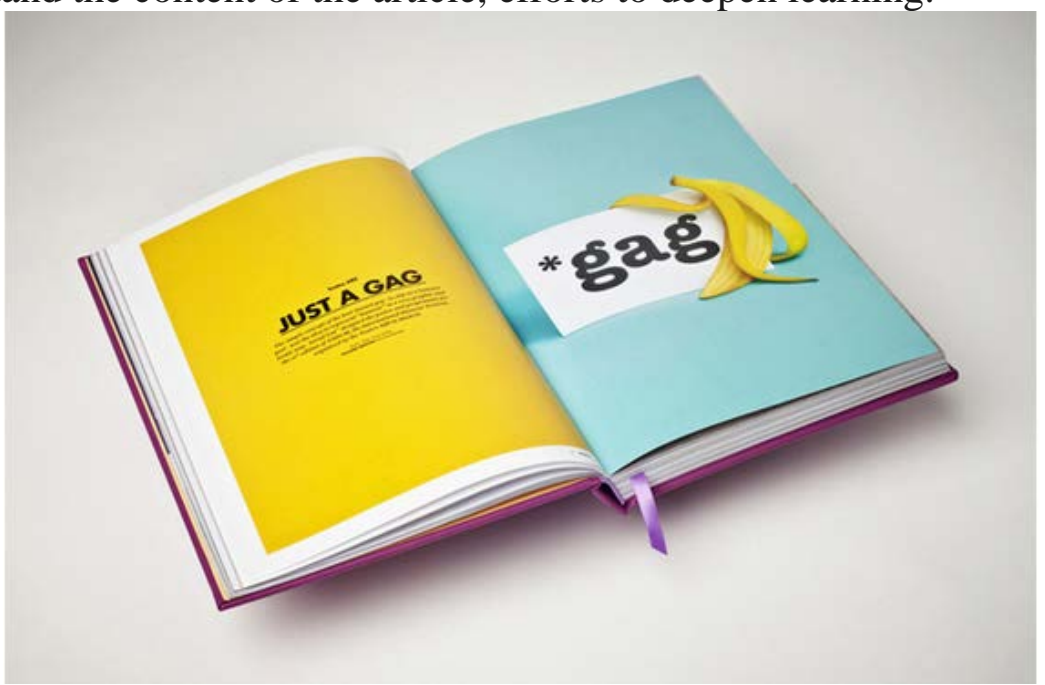

Books, paper selection of humanization. With advances in technology, the technology continues to develop, the main material of modern book design for paper, silk, cloth, leather, wood, 
fiber, metal, plastic and so on, different components of these materials, properties and texture, giving the perception and feel different. Books texture can give the reader a good visual experience, it is possible to some extent, so that the reader fall in love with this book, which will help promote the book. Books in the book promotion on packaging, occupies an important position, showing deep cultural heritage, people can not help but want to continue to the top of inquiry. Selection of books on paper, combined with modern science and technology, allowing readers to get a good reading experience. Now living high-paced, stress is the overall meaning of culture, books, our lives will be indispensable was factor and meticulous design concept, thoughtful reader interaction, allowing sales of books to reach a certain height, books, paper selection should constantly humane, refined.

\section{Conclusion}

Chinese book design in the 1990s experienced a transformation from concept to technology, the role of the book designer has also undergone a superposition of multiple identities, in this period, as the people's spiritual books and cultural products, at the time of the transformation of society, it has become the preferred choice of culture. Multidimensional space form book design and performance all the way to the humanities factor as the major factor, we see the key issue is to show change-related elements, the factors important cultural change presented above, it is possible to see the cultural important factors connotation transition above.

Book design to be a comprehensive one to take into account changes in the cultural transformation of the important factors, the cultural changes of the important reasons above, to achieve cultural change above important factors involved to achieve significant change in the culture of the above participatory approach prospective outline.

Way of book design and performance of multi-dimensional spatial form of participation to a number of factors, suitable for readers of different ages, different social experiences, different living environment departure, book design to diversify, rationalize. So in front of the school during the harvest knowledge, and enjoy the spirit of the harvest, the visual sense of shock.

Respect show multidimensional space form and manner of expression of the book design, presented a different culture, how to make modern readers get a good reading enjoyment, the only and content of the article, but also on and book design of this the book designer will need continuous efforts, for the time constant of the process.

\section{References}

[1] Shi Jiangying. Book design forms and manifestations multidimensional space research [D]. South China University of Technology, 2012.

[2] Gong Xiaoqing. Graphic design elements of visual representation of multidimensional [J]. Art Science and Technology, 2016 (2).

[3] Su Haoxu. graphic design elements - visual images of multidimensional performance studies [D]. Shandong University, 2009.

[4] Li Bochao, Di Xiaosong. Dimensional graphical representation in the form of three-dimensional space - On the concept of the book design [J]. Art Education Research, 2012 (4): 44-45.

[5] Li Bochao. Two-dimensional graphical representation in the form of three-dimensional space [J]. Changchun University of Technology, 2013.

[6] Liu Kai. Graphic design elements of the multidimensional visual representation [J]. Art and Science, 2015 (9).

[7] Wang Huanhuan. Multidimensional way of reading books design [D]. Beijing Institute of Printing, 2015.

[8] Zhao Huixin. Fun children's book cube shape design [J]. Science and Technology and published in 2012 (8): 69-71. 
[9] Chi Xi. Diversified book design art research [D]. Hunan University, 2011.

[10] Yang Yixin. Concept of book from book design to remodeling [J]. Popular Literature: Academic Edition, 2013 (13): 147-147. 